\title{
Voyage en train et paysage
}

\section{Karen Bowie et René Thom}

\section{OpenEdition}

\section{Journals}

Édition électronique

URL : https://journals.openedition.org/rhcf/1036

DOI : 10.4000/rhcf.1036

Éditeur

Rails \& histoire

Édition imprimée

Date de publication : 1 juin 2008

Pagination : 293-310

ISSN : 0996-9403

\section{Référence électronique}

Karen Bowie et René Thom, "Voyage en train et paysage ", Revue d'histoire des chemins de fer [En ligne], 39 | 2008, mis en ligne le 01 juin 2011, consulté le 22 avril 2022. URL : http:// journals.openedition.org/rhcf/1036 ; DOI : https://doi.org/10.4000/rhcf.1036 


\section{DOCUMENT}

\section{Voyage en train et paysage}

Karen Bowie a choisi le texte de cette communication du mathématicien et philosophe René Thom (1923-2002), prononcée lors du colloque "Arts et chemins de fer", $3^{e}$ colloque de l'AHICF réuni en novembre 1993 dont les actes ont été publiés par la Revue d'histoire des chemins de fer 10-11 (1994), pour sa valeur de référence et d'actualité dans notre analyse des paysages créés par le chemin de fer. On voit ici que le paysage dessiné par l'infrastructure ferroviaire et celui créé par la vision du voyageur qui regarde par la fenêtre du train ne sont pas opposés, mais au contraire très profondément liés.

Nous avons conservé la transcription du débat qui avait suivi cette communication, auquel a participé Jacques Guillerme (1927-1996), autre intervenant à ce colloque dont une partie de l'cuvre a été récemment rééditée*.

\footnotetext{
* Jacques Guillerme, L'Art du projet. Histoire, technique et architecture, Mardaga, 2008.
} 


\section{PAR LES FENÊTRES DU TRAIN. LA NOTION DE RÉFÉRENTIEL APPLIQUÉE À L'ART DE VOYAGER PAR LE TRAIN RENÉ THOM}

Dans un texte de souvenirs d'enfance «Songeries ferroviaires» [Thom, 1990], j'ai évoqué toute la fascination que dans mon jeune âge le chemin de fer a exercée sur moi. Ici je m'efforcerai d'expliquer ce qui, dans ce qu'on peut raisonnablement appeler mon vieil âge, subsiste de cet enchantement primitif. J'ai donc pris comme thème de ma communication le « voyage en chemin de fer », une activité que je continue à apprécier grandement bien que les progrès de la technologie moderne en aient modifié considérablement le caractère. Il nous faudra bien évoquer comment le train a changé au cours de ce siècle ${ }^{1}$ !

Du temps de ma prime enfance, vers 1925, automobiles et camions étaient encore rares et le chemin de fer avait alors un quasi-monopole du transport terrestre. Mais, dès le premier quart de ce siècle, l'impact du transport automobile sur route se fit sentir. Plus flexible, évitant les ruptures de charge, le nouveau transport avait beaucoup d'avantages. Les premières victimes de cette concurrence

1- Cette communication a été présentée une première fois au colloque réuni à Firminy en septembre 1992, à l'occasion du centenaire de la naissance d'André Chapelon. furent les lignes et réseaux de chemins de fer secondaires, lignes pour la plupart à écartement réduit et dont la longueur n'excédait guère la traversée de deux ou trois cantons. J'ai été très conscient de cette disparition dont on parlait beaucoup en famille lors de la mort des compagnies locales de tramways. Un peu plus tard, à l'époque où j'étais collégien, je rêvais d'écrire une thèse intitulée : "Grandeur et Décadence du chemin de fer d'intérêt secondaire»... Je ne sais si une telle thèse a été faite depuis et je me demande si, en France, il existe une institution centralisant toutes les informations sur toutes ces petites compagnies qui disparurent entre 1925 et 1940. Il aurait été bon qu'en un seul endroit on réunisse toutes les données concernant, pour ces compagnies, leur naissance (les circonstances politiques, économiques, administratives de leur création), le tracé des lignes, le matériel ferroviaire utilisé, les horaires décrivant leur activité jusqu'à leur disparition et, si possible, une iconographie relative à 
chaque compagnie aussi riche en documents photographiques que possible. C'est sur ce dernier point, je crois, qu'une institution serait bien nécessaire car c'est seulement en ouvrant les archives de famille locales qu'on peut espérer recueillir des documents photographiques sur l'activité de ces compagnies : une telle prospection ne serait pas chose facile à faire systématiquement. Si j'évoque ici le destin mélancolique de ces petits trains, c'est que, à beaucoup d'égards, ils figurent certainement parmi les premiers cas où l'évaluation du plaisir ferroviaire puisse se faire sans devoir l'accompagner des restrictions mentales inévitables lorsqu'on entre dans l'époque moderne. Après la mort des réseaux secondaires (si je ne me trompe, leur nombre actuel en France - compagnies touristiques exceptées n'excède pas celui des doigts d'une main), ce sont les lignes secondaires des grands réseaux (le Nord, l'Est, le PLM, le PO, etc.) qui, de 1930 à nos jours, seront remplacées par l'autobus. Or, nous le verrons, le plaisir du voyage en chemin de fer exige de n'être pas pressé. Ce qui fait que les amateurs du voyage ferroviaire pur devront, s'ils veulent trouver matière à leur faim, se diriger vers les pays du Tiers ou du Quart-Monde. C'est là qu'ils pourront trouver les meilleures occasions de satisfaire leur passion. Là où il y a peu de routes (et ample population), on trouve de ces trains bondés et pittoresques où, à chaque arrêt, le train subit l'assaut d'une nuée de vendeurs locaux de nourriture et de boissons. Ce n'est guère qu'au Mexique et au Pérou que j'ai eu l'occasion de pratiquer ce genre de trains dont l'intérêt humain se double souvent de la traversée d'une nature grandiose. Par contre, il ne faut pas ici être exigeant sur l'exactitude des horaires, ni sur le niveau des «accomodations » hôtelières. Bien entendu, il se trouvera peut-être parmi vous des esprits réalistes pour me dire qu'avant tout le chemin de fer est un instrument de transport et que notre époque est celle des « flux tendus ». Je me garderai bien de nier l'importance essentielle de la vitesse pour la rentabilité du train confronté à l'automobile, au camion, et à l'avion. Mais vous me permettrez d'abord de parler en esthète oublieux des priorités économiques. Je dirai plus tard comment ruser pour sauver quelque agrément d'un voyage en TGV...

D'où vient le plaisir que nous donnaient les petits tortillards de nos campagnes ? Je crois que, pour l'expliciter, il nous faut un peu de psychanalyse... Ce plaisir ne vient pas seulement de la contemplation des paysages (s'il y en a), ni de la fraîcheur de la campagne française (car, après tout, la France, de par sa terre et son climat, a l'une des plus belles campagnes du monde). Avant tout, il y a une identification de notre propre corps au véhicule qui nous loge, au convoi qui nous transporte et, pour finir, à tout le voisinage que nous pouvons percevoir ou même seulement concevoir en 
tant que carte mentale de notre environnement. Le mouvement du train réalise une sorte d'expansion graduelle du moi, suivant la cascade des référentiels emboîtés (voir annexe) EGO $\rightarrow$ compartiment $\rightarrow$ wagon $\rightarrow$ convoi total $\rightarrow$ voie $\rightarrow$ environnement

Mais le plaisir, s'il vient essentiellement de cette dilatation du moi, change de nature et d'intensité selon le stade où s'arrête l'identification. D'où une gradation naturelle des facteurs de plaisir et ce changement de référence est lui-même un plaisir.

\section{IDENTIFICATION AVEC LE WAGON}

Les premiers degrés de la cascade siège, compartiment, wagon ne sont pas sans importance, évidemment. Le problème du siège, de la place à occuper, du confort général de la voiture sont des facteurs bien connus et qui, pour certaines personnes, jouent un rôle considérable. Dans l'optique non commerciale qui est la nôtre actuellement, le problème du confort n'est pas essentiel. Beaucoup préféreront la ségrégation locale qu'imposaient les anciens compartiments à la disposition actuelle (couloir central de nos présentes voitures (Corail) : il est de fait que selon cette disposition, il est beaucoup plus difficile d'entretenir une conversation particulière avec audience limitée. Pour un voyageur celui que j'envisage - soucieux essentiellement de tirer profit de son voyage, le choix d'une place - qu'il est bon de prévoir variable - est aussi important - j'expliquerai plus tard pourquoi. En ce qui concerne le confort du roulement, les secousses transmises de la voie, on a fait de très grands progrès techniques (à la fois pour les rails et la suspension) mais c'est là un facteur qui intéressera surtout le voyageur soucieux de repos (ou tenant à faire une tâche personnelle, comme lire ou écrire) ; notre voyageur esthète n'y attachera pas tellement d'importance ${ }^{2}$ : on peut plaquer efficacement une mélodie endogène sur le rythme des secousses du rail. Récemment, à bord d'un express arrivant gare de Lyon, j'écoutais,

2- Parmi les compagnies de chemin de fer touristiques opérant actuellement en France deux des plus spectaculaires sont: Le Chemin de fer (ex-minier) de La Mure (Isère) et le Chemin de fer forestier d'Abreschwiller (Moselle). Dans les deux cas, la vitesse est très lente et le matériel extrêmement grinçant, ce qui ne nuit pas au pittoresque. 
à l'entrée d'un soufflet, la vibration caractéristique - par effet différentiel émise par les voitures lorsqu'elles parcouraient - à l'entrée des quais - les courbes des raccordements entre deux aiguillages. Et je me disais que le compositeur Pierre Henry aurait pu tirer de cette suite de sons une pièce magnifique de musique " concrète ». Ces défauts sonores sont en fait des indices qui permettent souvent une meilleure identification d'EGO avec le convoi. Dans un tunnel de crête on peut, à l'audition du bruit des rails, discerner si le train se trouve encore en montée ou commence à descendre de l'autre côté de la montagne. J'en viens maintenant aux identifications de dernier degré, avec le convoi, avec la voie, avec l'environnement.

\section{IDENTIFICATION AVEC LE CONVOI}

Dans cette extension notre corps se trouve inclus, comme un infime parasite, au sein de ce grand organisme, le convoi. Un imaginaire implicite (peut-être commun à beaucoup d'êtres vivants) assimile le convoi à un de ces animaux découpés en segments par métamérie, comme la chenille, le lombric, le mille-pattes. Chaque article de la structure est en ce cas un wagon. Et comme les articles de l'animal métamérique peuvent suivant leur position sur l'axe céphalo-caudal acquérir différentes spécialisations fonctionnelles (comme la tête, les gonades, l'anus..., etc.), le train présente en général une structure canonique de la tête à la queue : locomotive, (autrefois) tender, fourgon de tête, voitures voyageurs, fourgon à bagages, il se terminait dans les temps anciens par une voiture serre-frein (la caboose des trains du Far-West). Le langage a - je suppose universellement - imposé en technique ferroviaire la métaphore tête-queue du convoi (étendue au quai dans la formule célèbre : "Au bout du quai les ballots!»). Pour certains tracés de voie particulièrement tortueux on peut parfois d'une fenêtre apercevoir une bonne part du convoi, ce qui visuellement réalise une identification partielle parfois hautement jouissive. Dans cette identification, notre âme individuelle cherche un correspondant. Elle le trouve dans ce cerveau qu'est la cabine de commande, en fait dans la personne du conducteur du train. Bien que la hiérarchie standard connaisse un chef de train, je suis convaincu que le conducteur est plus important dans l'imaginaire du voyageur, car ce dernier joue un rôle essentiel dans la collision virtuelle qui peut affecter le train en mouvement avec un obstacle fixe ou mobile. C'est l'équivalent du pilote de l'avion. Dans les trains modernes, le conducteur peut nous donner par haut-parleur des informations sur les incidents de route (arrêts inopinés, pannes, incidents divers d'origine interne ou externe, etc.) et il le fait, le plus souvent, avec beaucoup de serviabilité. On ne s'étonnera pas si les contacts entre voyageurs et conducteur sont si rigoureusement codifiés. 
Car l'intrusion malencontreuse du désir d'un voyageur dans la marche du convoi peut avoir de funestes conséquences. Qu'on se rappelle le récent accident de la gare de Lyon, causé par une suite d'événements où, à l'origine, on trouve une voyageuse tirant la sonnette d'alarme pour arrêter son train à une station non prévue à l'horaire et, à la fin, un conducteur prenant le départ de son train à l'arrêt avec quelques minutes de retard. Je ne crois pas qu'on puisse, en général, accuser les autorités de minimiser leurs difficultés pour obtenir la patience des usagers lors de pannes. S'il m'est arrivé de pester dans un train à la suite d'arrêts inexpliqués, la cause en était en général des perturbations politiques (manifestations, barrages de la voie, etc.) extérieures à la SNCF et les promesses mensongères du haut-parleur n'y prirent jamais la même ampleur que les déclarations des compagnies aériennes lors d'une panne de leur appareil.

IDENTIFICATION AVEC LA VOIE

Le convoi suit la voie. Il est donc normal pour un passager de s'intéresser au tracé de la voie. En faisant d'abord abstraction du relief (la verticale, troisième dimension), la voie est une courbe plane. Comme il est bien connu que la ligne droite est le plus court chemin d'un point à un autre, on doit penser que si la voie est courbe en un point, c'est qu'il y a de sérieuses raisons pour qu'il en soit ainsi. Un des grands motifs de réflexion, dans le voyage en chemin de fer, est la justification du tracé de la ligne. Mais avant d'aborder ce problème il faut observer que la voie comporte aussi d'autres singularités que les courbes. Il y a, en particulier, tous ces dispositifs qu'on appelle appareils de voie: aiguilles et signaux en général. Ils constituent autant de points singuliers du tracé (par point singulier, j'entends ce terme au sens mathématique : un point singulier est un point qui n'est pas comme les autres). Les arrêts en dehors des gares sont en général dus à la fermeture de signaux. Le propre de la voie est de signifier un chemin imposé (une chréode, comme disait le biologiste anglais Waddington) mais il reste un élément de liberté pour le parcours, c'est le choix de la branche de la bifurcation offerte par une aiguille standard abordée par la pointe. Il n'est pas usuel de laisser au conducteur d'un convoi le choix de la direction à prendre lorsqu'il rencontre une aiguille par la pointe (c'est l'autorité globale qui en principe le décide et oriente l'aiguille sur la voie désirée) ; mais j’ai vu des tramways (urbains) capables d'agir sur l'aiguille qu'ils abordent par commande électrique (à Strasbourg autrefois et actuellement à l'étranger). En arrêt dans les gares, pour réaliser une correspondance, le voyageur pourra parfois être impliqué dans des manœuvres affectant son wagon. Il ne manquera pas alors d'observer attentivement la manœuvre en s'efforçant 
de la prévoir. L'aiguille, au fond, c'est la manifestation de la liberté humaine au sein de ce monde algébriquement fixé qu'est le schéma global des voies. C'est pourquoi, très fréquemment, c'est par l'aiguillage qu'apparaît l'accident. On me pardonnera ici d'introduire cette auto-citation : « La bifurcation engendre la catastrophe... » La catastrophe (relativement récente) de la gare d'Ay dans la Marne en est une tragique illustration. Il est certain que le trafic ferroviaire externe - dans la mesure où il existe - est un élément du plaisir du voyageur. Croiser un train (surtout sur une ligne à voie unique) est toujours un élément de distraction. Mais nous n'irons pas jusqu'à la passion de ces collectionneurs anglais que j'ai vu noter sur un carnet de route tous les numéros des locomotives croupies au fond des dépôts et des wagons de marchandises dormant sur des voies oubliées qu'il leur arrive d'apercevoir. Mais un petit sport non négligeable consiste, en présence d'une voie de garage parallèle à celle du train, de parier pour la dégradation progressive de cette voie sous les arbustes et les herbes folles ou au contraire pour sa réadmission correcte sur la voie principale.

\section{LA VOIE ET LE TERRAIN}

Revenons donc à la voie et à ses courbes. Pour comprendre le tracé de la voie il faut revenir à sa fonction primitive : assurer une connexion entre deux points (A) et (B) considérés comme économiquement importants, par exemple deux villes. Le jour où les planificateurs ont considéré le problème de déterminer le tracé, il est fort rare que le segment rectiligne $\mathrm{AB}$ puisse être considéré comme acceptable. Il peut y avoir contre ce tracé des objections dirimantes, soit liées à des obstacles naturels : reliefs, profondes vallées, pièces d'eau (lacs ou grands fleuves), soit liées à des implantations humaines préexistantes: agglomérations rencontrant $\mathrm{AB}$, ou voisines de $\mathrm{AB}$, industries à desservir, etc. En règle générale, on déterminera un certain nombre de points à desservir $\mathrm{Xj}$ intermédiaires entre $\mathrm{A}$ et $\mathrm{B}$, lesquels pourront être ordonnés de telle manière qu'on aille de $\mathrm{A}$ en $\mathrm{B}$ par une suite de trajets $\mathrm{XjXj}+\mathrm{I}$ qui ne se rencontrent pas (sur la carte). Il vaudrait évidemment mieux que la direction $\mathrm{XjXj}+\mathrm{I}$ pour tout $\mathrm{j}$ ne soit pas trop différente de la direction globale $\mathrm{AB}$, de manière à minimiser la longueur du trajet global. Ce choix préliminaire des points à desservir est en général le résultat d'une longue confrontation d'intérêts politiques, économiques, techniques qui n'est pas ici de mon propos. Une chose y est certaine : en voulant contenter tout le monde, on arrive en général à une mauvaise solution. Rattacher une ville négligée au tracé définitif par un embranchement est en général à déconseiller (qu'on pense au malheur de la ville d'Amiens, négligée par le tracé du TGV Nord). Le fameux plan Freycinet qui, au début de la III République, se proposait de mettre 
une gare à moins d'une journée de cheval de tout point de la France continentale procédait sans doute d'une louable intention, mais ne pouvait conduire qu'à des projets économiquement aberrants; car ce que la voie doit concrétiser, ce sont des flux et non des aires.

Quoi qu'il en soit, nous supposerons fait ce choix des points intermédiaires et nous nous trouvons ramenés au problème initial où seules les contraintes géographiques (relief et cours d'eau) sont à prendre en compte. Au début, la contrainte du relief pesait d'un poids relativement lourd car la relative faiblesse de la force de traction d'une locomotive isolée exigeait un coefficient d'adhérence des roues considérable (ceci pour un convoi long et lourd), ce qui limitait la pente des rampes admissibles à quelque 10 pour mille (on allait jusqu'à 30 pour mille dans le cas des lignes de montagne, en limitant la longueur des convois). Ces contraintes ont pesé lourd dans le tracé des lignes classiques. Nous laisserons ici de côté le cas du TGV où l'on s'est inspiré de la technique des autoroutes ; déjà, en 1910, on avait construit des lignes de tramways de montagne où la pente s'élevait à $8 \%$ (le convoi étant réduit à une motrice et une remorque). Cette différence technique a de grands effets sur l'attitude de notre esthète qui, en TGV, aura peu à se mettre sous la dent. Car la sensibilité au relief est l'un des éléments majeurs du sentiment d'appartenance au paysage. Par suite de cette infirmité des trains à ne tolérer que de très faibles rampes, le tracé de la voie en tout point ne peut trop différer d'une ligne de niveau. Or comme le montrent à l'envi nos cartes IGN d'échelle standard, en régions à relief varié les lignes de niveau sont loin d'être rectilignes : elles peuvent présenter des accidents angulaires passablement rugueux. D’où la nécessité des courbes pour corriger les discontinuités angulaires, la nécessité de travaux pour régulariser le tracé. Heureusement la taxonomie de ces écarts de niveau est vite faite : s'il s'agit d'un gain en hauteur, on aura une voie en remblai ; s'il s'agit d'abaisser le niveau, on aura une tranchée si la différence de niveau n'excède pas une vingtaine de mètres, au-delà on envisagera un tunnel. On s'efforcera ensuite, pour minimiser le déplacement de terre, d'équilibrer remblai et déblai, un vieux problème qui préoccupait déjà les ingénieurs en voirie du XVIII siècle. Enfin nous mentionnerons la présence éventuelle d'ouvrages majeurs, comme les ponts (ou viaducs) franchissant de profondes dépressions et les tunnels franchissant une zone trop surélevée pour une tranchée. D'un point de vue de pure mathématique, le choix d'un tracé à extrémités données est un problème variationnel avec conditions d'inéquation portant sur les dérivées premières (la pente de la voie); en fait ce problème compte peu en regard des contraintes d'origine humaine: politiques, économiques et financières. 
Tout est possible si on accepte d'y mettre le prix. Mais il n'empêche que le problème " géométrique » du tracé reste sous-jacent et l'intuition que le voyageur en a est un des éléments « esthétiques » majeurs du voyage en chemin de fer. Le bon voyageur est celui qui saura se replacer dans l'optique de ceux qui ont tracé la ligne.

Dans le chemin de fer de montagne proprement dit (comme il s'en trouve en Suisse), le problème se complique du fait de la présence de segments à forte rampe munis de crémaillère. Comme le relief est considérable, on accepte des moyens spécifiques pour le vaincre. En France, nos cours d'eau n'ont que bien rarement cascades et rapides - sauf en montagne et proches de leurs sources. De là résulte que la plupart des vallées sont en pente faible. Or une vallée est de même forme qu'une tranchée (à flancs relativement doux, avec un fond plat large ou étroit). Dans la mesure où le cours d'eau ne méandre pas, il est de règle générale que la voie emprunte une vallée. Si le profil de la vallée est approximativement un $\mathrm{V}$, la voie devra être établie en corniche, côté gauche ou côté droit. Il en résulte pour le voyageur soucieux du paysage une dissymétrie flagrante : si, dans le sens de la marche, la voie est sur le flanc gauche de la vallée, la fenêtre gauche du wagon ne permettra guère que l'observation du minéral dont est faite la paroi qui limite à gauche la plate-forme de la voie. La fenêtre droite, par contre, permettra une vue plus intéressante car elle domine le ravin où s'écoule la rivière. C'est là, évidemment, qu'ira s'installer notre esthète. Ce caractère dissymétrique des vues est malheureusement la situation générale. De là résulte qu'on ne peut faire un voyage intéressant en chemin de fer que si la densité des voyageurs est suffisamment faible pour qu'on puisse sans gêner autrui « voltiger » d'un côté à l'autre du wagon. Une situation qu'on trouvera rarement en TGV, mais qui est fréquente dans les autorails campagnards comme le Cévenol (Nîmes - Clermont-Ferrand) pour le Massif central, ou Dole - Saint-Claude pour le Jura. On recommandera aux amateurs de pratiquer ce type de lignes. Qu'ils se hâtent car les soucis actuels de rentabilité de la SNCF font craindre qu'elles ne disparaissent bientôt ! Même si le $\mathrm{V}$ de notre profil de vallée est très arrondi, cet effet de dissymétrie est toujours présent. Si le cours d'eau cesse d'être rectiligne, il se peut que la ligne change de rive grâce à un pont enjambant la rivière. En ce cas, il y aura intérêt à changer de côté dans le wagon. Si le fond de vallée est relativement plat, et que le cours d'eau méandre, il n'y aura pas très grand intérêt à changer de place ; par contre on pourra s'efforcer de deviner, au prochain pont, en quel sens coule la rivière (sur la ligne Paris-Strasbourg, en regardant la Marne, on peut jouer à ce jeu entre Paris et ChâteauThierry). Ce qui structure une ligne, finalement, c'est le nombre des bassins de 
rivières qu'elle doit emprunter. En ce sens, il faut s'efforcer de « vivre » les changements de bassins et ceci d'autant plus que le franchissement d'un seuil séparant les bassins est en général marqué par une tranchée, un tunnel dont les parages offrent des courbes et/ou des dénivellations marquées. Dans certains cas (comme pour le tunnel de Blaisy-Bas sur ParisDijon), la localisation de la crête entre Seine et Saône ne fait aucun doute. Dans d'autres, la détermination du seuil est beaucoup plus douteuse. Le franchissement d'une tranchée peut signifier changement de bassin comme il peut signifier persistance du bassin. Pour lever l'ambiguïté il faut, à la vue du cours d'eau qu'on retrouve ensuite, déterminer le sens de l'écoulement. Cela peut être difficile à simple vue. Il faut alors se fier à cet axiome des géographes (ou géomorphologues) disant qu'en un confluent du type Y, le sens de la branche opposée à l'angle aigu du confluent est le sens « descendant», axiome qui demande une interprétation qu'il peut être difficile de donner si le confluent n'est vu que dans une durée très brève. Certaines portions de ligne sont d'interprétation difficile. Par exemple : ayant dû emprunter souvent la ligne Paris-Limoges, le tronçon compris entre Argenton-sur-Creuse où la ligne franchit la Creuse d'Est en Ouest - et Limoges m'a souvent posé des problèmes d'interprétation que je n'ai pu résoudre qu'à l'aide d'une carte routière. Il s'agit là (jusqu’à La
Souterraine) d'un tronçon en crête entre deux bassins, gagnant progressivement en altitude mais oscillant entre des bassins limitrophes (S-O ou $\mathrm{N}-\mathrm{E})$; après La Souterraine, un tunnel de crête nous mène dans le bassin de la Gartempe où la voie redescend en empruntant aussi une arête descendante assez aiguë qu'elle franchit à plusieurs reprises. Du viaduc de la Gartempe, la voie remonte sur la rive gauche jusqu'en gare de Saint-SulpiceLaurière d'où un tunnel de crête sous les monts d'Amblazac nous amène dans le bassin de la Vienne et l'on descend sur Limoges en enjambant quelques affluents secondaires. Au fond, une connaissance complète de la géographie d'une ligne ne s'acquiert que progressivement. C'est le travail que les conducteurs doivent faire quand ils sont mis sur une ligne qu'ils n'ont jamais pratiquée. Pour un voyageur, il faut beaucoup d'obstination pour acquérir une mémoire complète de tous les accidents d'une ligne et, même avec beaucoup d'expérience, on peut à chaque nouveau voyage apercevoir des détails non remarqués auparavant (existence d'une combe brièvement entrevue, d'un vallon plaisant, d'une ruine de château fugitive, d'un village non remarqué auparavant). C'estl'enregistrement de tous ces détails qui font le plaisir du voyage, plaisir beaucoup plus complet si on a pu les intégrer dans un schéma global de l'environnement de la ligne. Finalement, il me semble correct d'affirmer que c'est la troisième dimension, le 
relief, qui est le facteur essentiel d'intérêt d'un tracé. Une ligne en terrain plat, rectiligne, n'a que sa monotonie à offrir. Dans les affleurements des tranchées, on peut deviner la nature géologique du sol et ainsi en tirer des éléments de localisation. C'était la méthode préconisée par C.-A. de Lapparent dans son ouvrage classique sur l'emploi de signes géologiques pour le trajet Paris-Dijon. Mais il faut une culture considérable pour interpréter correctement ces signes et l'erreur est facile. Sur la ligne Dijon-Lausanne, par exemple, le voyageur traverse la plaine haut-saônoise rapidement, franchit la Saône à Auxonne et, deux kilomètres avant Dole, rencontre une tranchée assez profonde qui se termine par un tunnel assez court (le tunnel de Champvans). Il pensera légitimement qu'il s'agit là d'un premier contrefort de la chaîne du Jura, calcaire comme il se doit... Erreur, il s'agit d'un lambeau de terrain granitique primaire, la montagne de la Serre émergeant entre la vallée du Doubs et celle de l'Ognon (mais l'erreur est bénigne car, probablement, sans la secousse tertiaire du Jura, le lambeau granitique de la Serre n'aurait pas émergé des sédiments qui le recouvraient). La sensation du relief est évidemment liée à l'effort nécessaire pour le vaincre. Ainsi le cycliste, qui sait ce que signifie une côte (et une descente), a un sens du relief beaucoup plus accusé que l'automobiliste, pour quil l'effort personnel se limite à changer éventuellement de vitesse. C'était un rare privilège de la locomotive à vapeur que d'avoir par le régulateur une gamme continue de changements de vitesse, ce qui, à l'audition de son halètement, permettait de se représenter très précisément l'effort de la machine. En traction non autonome, comme la traction électrique, on perd ce sentiment du relief, et il faut une observation attentive pour reconnâtre si la voie monte ou descend; dans les autorails campagnards évoqués plus haut, le changement de vitesse est un changement de fréquence du ronflement du moteur aisément perceptible. Le folklore ferroviaire évoque de nombreux cas où, ayant épuisé sa provision de vapeur (ou le foyer n'en fournissant pas assez), la locomotive « calait » et le convoi s'arrêtait (voire redescendait). J'ai connu un petit train touristique, en Australie, nommé « Puffing Billy » (dans la banlieue de Melbourne), où on offre au client passager l'agrément d'un tel arrêt en pleine côte, en pleine forêt d'eucalyptus. Sur notre réseau national, je n'ai connu qu'une fois une panne de ce genre, dans des conditions assez exceptionnelles il est vrai. C'était en 1944, le débarquement anglo-américain venait d'avoir lieu et, de Paris où j'étais normalien, j’avais décidé de regagner ma famille dans l'Est. Je pris donc un train régulier pour Belfort qui partait alors de la gare de la Bastille (le viaduc de Nogent-sur-Marne étant coupé par des bombardements). Nous prîmes donc la voie empruntée par l'actuel RER A jusqu’à Boissy-Saint-Léger et, 
au-delà par la forêt, en direction de Verneuil-l'Etang où l'on rejoignait la grande ligne de Troyes. Mais à la localité de Villecresnes, située dans un creux, notre machine ne put venir à bout de la rampe qui remontait sur le plateau. On dut faire venir de Paris une machine de renfort, laquelle - en nous « poussant au cul »- réussit à nous remonter au niveau du plateau de Brie-Comte-Robert. Ce voyage risqué n'eut pas d'autre incident.

Pour en revenir aux sources du plaisir dans le voyage en chemin de fer, on pourrait se demander quel a été l'impact de l'automobile sur cette sensibilité. Beaucoup de clients du chemin de fer sont (ou ont été) des automobilistes. Évidemment, le voyageur du train est passif, il n'a aucun contrôle sur le mouvement de son véhicule contrairement à l'automobiliste qui peut croire qu'il est à l'origine du mouvement de sa voiture. Mais, l'automobiliste finira par s'en rendre compte, sa liberté est strictement limitée. Rappelons ici que les feux de carrefour sont historiquement un décalque de la signalisation ferroviaire (apparus aux États-Unis vers 1920) et la limitation de vitesse y est également commune. De plus, le peu de liberté qui reste au conducteur engage strictement sa personnalité. En sorte que si l'on veut garder une certaine disponibilité pour le paysage, pour l'environnement, on finit par soupirer d'être en train. Il reste à l'automobiliste une supériorité, celle du choix de son itinéraire. Mais cette liberté tend à se restreindre. L'effroyable densité des bouchons les jours de grand trafic semble montrer que, soit les automobilistes considèrent qu'ils n'ont aucune possibilité de varier leur itinéraire, soit qu'ils ont la paresse de chercher une variante. Et puis il y a la compétition des poids lourds. Stendhal, vers 1830, avait posé cette question (je cite de mémoire) : " Que deviendront les capitaux investis dans les chemins de fer quand on aura trouvé le moyen de faire rouler les wagons sur les routes ? » On pourrait continuer : «Que se passera-t-il quand on aura trouvé le moyen de faire rouler les trains sur les routes?»

On voit en ce moment sur nos routes de tels attelages de poids lourds qu'on se demande pourquoi s'arrêter en si bonne voie. Un mode de transport ne peut-il pas périr de saturer l'espace?

Dans cette perspective, on pensera à se tourner vers la troisième dimension, encore non saturée. Mais il semble bien qu'un engin volant soit nécessairement d'un volume, d'une masse qui en compromettent un usage individuel. Ni les ULM, ni la bicyclette volante qui a permis de franchir la Manche ne peuvent prétendre à la généralisation pratique. L'avion en tant que mode de transport collectif est parfaitement au point et son taux d'accident n'est pas de nature à décourager les clients prospectifs, je me bornerai ici à reprendre mes considérations antérieures sur le voyage en chemin de fer dans le cas 
du transport aérien. Dans ce cas, on a pratiquement la suite des référentiels EGO $\rightarrow$ (Cabine) $\rightarrow$ Avion $\rightarrow$ Environnement

Ce qui frappe ici, c'est que l'environnement n'a pas de morphologie propre, la forme et la quantité des nuages sont essentiellement variables ; si l'on veut se repérer effectivement, il faut projeter le point choisi sur la surface terrestre, en y ajoutant l'altitude comme troisième coordonnée. Il est de fait qu'une des rares sources de plaisir du voyage en avion consiste - si l'on dispose d'un hublot adéquat et si la visibilité le permet à repérer les détails géographiques observables, à les identifier (éventuellement avec l'aide de la carte fournie aimablement dans le magazine de la compagnie) et à observer les changements dus à la progression de l'avion. Ceci se présente fréquemment, par exemple au-dessus de la mer adriatique où les côtes italienne ou dalmate peuvent souvent être suivies. Un autre cas intéressant est celui où la troisième dimension (la hauteur des montagnes) entre en jeu. Ainsi le survol des Alpes, souvent spectaculaire par beau temps dans le vol Paris-Milan. Dans le trajet Téhéran-Tabriz des compagnies iraniennes, l'avion se faufile dans un corridor montagneux menant à un col de l'Elbrouz qu'il survole à faible altitude. Dans le même ordre d'idées, l'atterrissage dans certaines villes est impressionnant. Ainsi Genève pour son lac, entre Jura et Alpes, ainsi
Hong-Kong où l'avion paraît frôler les gratte-ciels. Dans ce cas, une des composantes du plaisir est le sentiment d'un danger virtuel dont, non moins régulièrement, on triomphe. $\mathrm{Si}$ je me permets d'évoquer ici l'avion, c'est qu'il est de mon devoir de vous parler du voyage en TGV. Ici, avec la pleine vitesse du convoi, il n'est plus guère possible d'apercevoir les détails locaux (par exemple, la géométrie des confluents) dont j’ai parlé pour le train ordinaire. Le TGV n'est plus un train mais une fusée. Néanmoins, l'expérience révèle qu'on peut y déceler les pentes un peu fortes marquées par une variation de la vitesse. Pour la géographie il faut, pour se repérer, passer à une échelle plus grande des éléments de relief, se rattacher à des marqueurs plus gros et plus lointains. Par exemple, sur Paris-Lyon la traversée de la Forêt d'Othe entre «Sens » et le point de traversée de la ligne classique entre Saint-Florentin et Tonnerre est parfaitement décelable et on peut s'amuser à corréler ces impressions de relief avec certaines vues typiques du voisinage. De même la traversée de la dépression d'Avallon où je cherche toujours la trace de moins en moins visible de l'ancienne ligne d'Avallon à Semur et Les Laumes qu'il m'arriva un jour, il y a un demi-siècle, d'emprunter... C'est cet égrènement de détails significatifs qui constitue à mes yeux l'essentiel de ce que le TGV peut nous offrir. Et comme cet éventail de détails restera toujours incomplet, tout voyage offre une possibilité de l'enrichir. Le 
TGV offre un survol mais néanmoins terrestre. En bonne logique, on aurait dû munir les sièges de TGV de ceintures de sécurité puisqu'on les impose aux avions et aux voitures individuelles. C'est peut-être que le chemin de fer offre, avec le référentiel du train global, un système suffisamment massif pour rendre toute collision avec un obstacle imprévu inoffensive. Mais ceci impose l'obligation du « site propre ", d'accès rigoureusement interdit à l'animal, à l'homme et à ses véhicules; c'est écologiquement une contrainte assez lourde. Qu'on est loin du tramway de notre enfance qui traversait les villages en empruntant les rues et rasant les maisons... On avait alors le sentiment d'un voyage qu'on aurait pu faire à pied. Le transport moderne nous assimile à un paquet qui perd tout contact avec le monde extérieur au départ et ne le retrouve qu'à l'arrivée. Seule l'imagination du chemin continu joignant l'origine à la fin, en restaurant à travers l'espace la continuité temporelle du moi, peut nous faire retrouver la vraie vie.

\section{ANNEXE}

\section{Sur la notion de référentiel}

Ce terme a reçu de la part du philosophe suisse Ferdinand Gonseth une interprétation qui généralise sa signification primitive de repère trirectangle. Comme cette notion a une origine ferroviaire, je ne crois pas déplacé ici de vous en citer l'origine, extraite du livre de Gonseth, Le Référentiel, univers obligé de médiatisation, Lausanne, L'Âge d'Homme, 1980.

« Il y a de cela bien des années, je montais avec ma famille par le train de Stansad à Engelberg. À l'avantdernière station notre wagon s'était arrêté devant un groupe de beaux sapins. Levant les yeux vers la fenêtre, je tressaillis de surprise : les sapins, de leurs troncs parallèles, semblaient barrer obliquement toute l'étendue de la vitre. Par quel maléfice les sapins ne s'élevaient-ils pas à la verticale dans ce pays comme dans les autres ? Je m'approchai de la fenêtre. Comme par enchantement le maléfice s'évanouit : ce que j'apercevais était un paysage normal, les sapins y respectaient parfaitement les normes de la verticalité. Avais-je rêvé ? Je revins en arrière et fis lentement du regard le tour du coupé, pour reporter ensuite toute mon attention sur la fenêtre. À nouveau les sapins la barraient obliquement. Mais à peine m'étais-je rapproché que déjà cette vision s'effaçait pour faire place à ce que je savais être la « réalité ». Ne me dites pas que j'avais été simplement la victime de ma mauvaise vue. À cette époque, ijy 
voyais encore assez pour ne pas être grossièrement abusé. Et d'ailleurs, preuve tout à fait décisive, il m'arriva de passer plus d'une fois par là et de voir le phénomène se reproduire.

Ceci s'explique aisément. C'est qu'à cet endroit, la voie n'est pas horizontale. Ce qu'à l'intérieur du coupé, je prenais pour des verticales n'était rien d'autre que des obliques. Or c'est dans ce cadre, dans ce contexte, dans ce référentiel que j'interprétais les impressions venues de l'extérieur. Si l'image de la vitre restait droite, c'est celle des sapins qui devait être penchée. Mais dès que je me rapprochais de la fenêtre, le cadre naturel dans son ensemble reprenait sa fonction de référentiel normal. »

II faut savoir que la ligne StansadEngelberg (actuellement Lucerne -Engelberg) est une ligne à voie métrique, à traction électrique, qui, pour l'essentiel de son parcours, opère par simple adhérence. Mais les derniers kilomètres avant Engelberg (1 $100 \mathrm{~m}$ d'altitude) sont équipés de crémaillère et présentent une pente avoisinant $30 \%$. C'est dans cette section, à l'arrêt dit Gruenenwald, que se présente le phénomène décrit par Gonseth. Dans un voyage récent, j’ai pu vérifier le phénomène, à cela près qu'à cet endroit les sapins se font rares. Par contre, on peut retrouver le phénomène - très frappant - sur l'apparente obliquité, à travers la vitre, des poteaux qui supportent les caténaires.

Gonseth a présenté une théorisation du phénomène grâce au concept, par lui introduit, de « référentiel». Sous forme essentielle le référentiel est un corps solide (idéal) contenant le corps du sujet et par rapport auquel le sujet évalue ses déplacements (ce serait, par exemple, le lieu aristotélicien - topos de la physique aristotélicienne car le lieu (topos) veut être immobile. Des discussions avec des physiologistes m'ont convaincu qu'une telle construction existe psychiquement même chez les animaux. Car, pour un animal, il est plus important d'évaluer ses déplacements par rapport à un système de repères considéré comme fixe que de se constituer un schéma mental du déplacement de son corps par rapport à lui-même (schéma corporel d'Henri Wallon). Les référentiels considérés ici doivent donc être considérés comme des cartes tridimensionnelles où EGO évalue ses déplacements.

Gonseth a introduit des généralisations philosophiques de cette notion qu'il serait tout à fait hors de notre propos de considérer ici. Ce phénomène des «sapins obliques » peut être pour l'essentiel modélisé par un schéma catastrophique relativement simple (celui, classique, de la fronce), dans l'esprit de celui offert par E.-C. Zeeman du conflit entre deux interprétations perceptuelles d'une même forme visuelle ambiguë. On peut admettre qu'à notre corps est associé par l'ontogenèse un repère euclidien $\Phi$ défini par les trois gradients « morphogénétiques » : haut-bas (alias céphalo-caudal) noté $\Delta$, gauche-droite (gd), dorsal-ventral (dv). Pour chaque 
position (p) du corps, il existe un homomorphisme $h(p)$ qui envoie ce trièdre originel $\Phi$ sur un trièdre euclidien approprié, un référentiel local approprié (R)p ; par exemple, si je suis couché sur le dos dans un lit étroit, l'image par $\mathrm{h}(\mathrm{p})$ de la verticale hautbas sera l'axe horizontal de mon lit, et l'image de l'axe dorso-ventral (dv) sera la verticale ascendante de (R). Du fait de la grande variabilité de cet homomorphisme $\mathrm{h}(\mathrm{p})$, qui varie pour tout déplacement global de notre corps, nous oublions le repère corporel originel $\Phi$ pour ne conserver que le repère euclidien Rp but de $\mathrm{h}(\mathrm{p})$ qui, lui, est en général imposé par les contraintes définies par les parois «verticales» de notre siège ou de notre chambre. Mais une fixation de longue durée du trièdre $h(p)$ en une position aberrante (par exemple renversant la verticale) ne serait pas sans créer de sérieux troubles physiologiques. C'est dire que bien qu'échappant parfaitement à notre conscience, le trièdre originel $\Phi$ n'en continue pas moins une existence latente, touchant surtout au maintien de la verticale : les directions horizontales, qui tolèrent une rotation autour de l'axe du corps, sont biologiquement moins marquées. Le morphisme $\mathrm{h}(\mathrm{p})$ a pour origine essentielle la vision de l'environnement, d'où la difficulté de se repérer dans l'obscurité.
Cela étant dit, revenons aux « sapins obliques ». Le phénomène peut être décrit comme un conflit entre deux déterminations de $\mathrm{hW} \Delta$. La première $\mathrm{hW} \Delta$ est celle liée au repère associé au wagon arrêté sur la voie en pente. La seconde hE $\Delta$ est le morphisme dicté par la vue sur l'extérieur, à travers la vitre de la fenêtre $(F)$. Le paramètre de contrôle de la transition sera, par exemple, l'angle solide s sous lequel l'observateur voit la fenêtre $(F)$, selon le schéma classique en "wiggle » (voir figure 1). La fenêtre (F) joue un

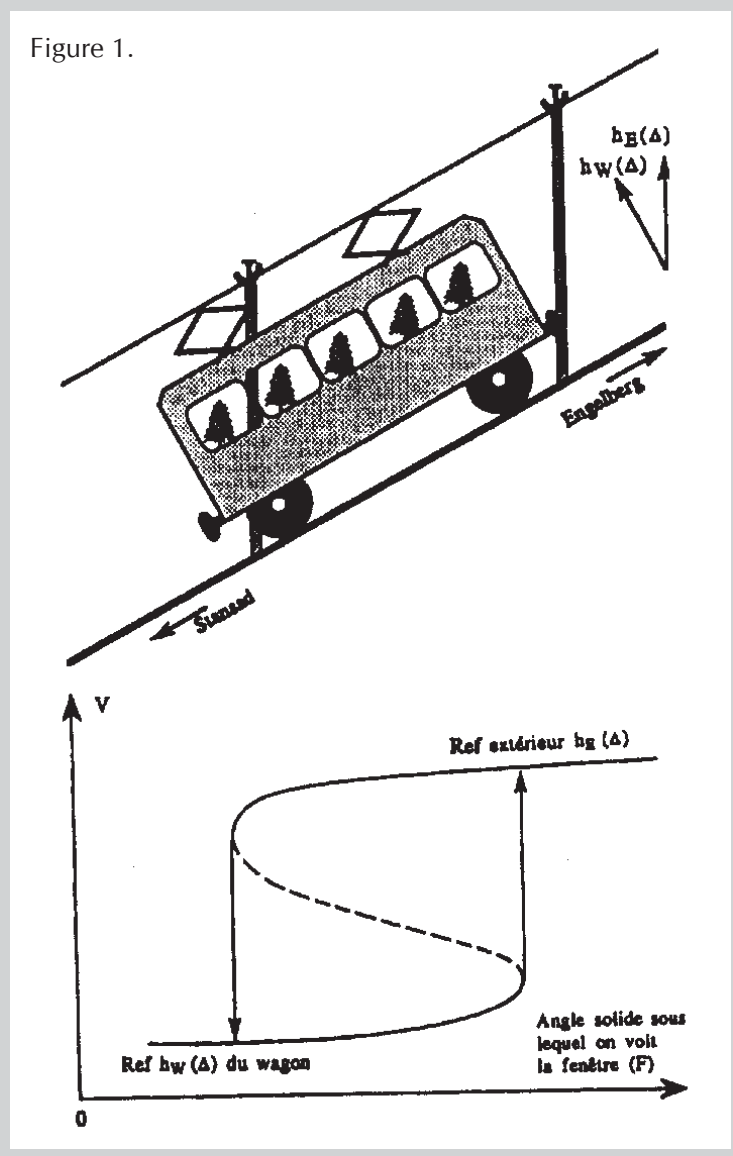


rôle central dans le phénomène ; c'est une lacune de la clôture du domaine (wagon) par laquelle va s'infiltrer la lumière porteuse de formes extérieures susceptibles d'être vues et, par suite, d'entrer en conflit avec la verticale du repère $h(W)$. On observera que la verticalité des troncs de sapin indépendante de la pente du sol est une connaissance acquise relativement tardivement : les expériences de Luquet sur le dessin enfantin montrent que chez l'enfant de moins de 5-6 ans c'est l'orthogonalité tronc-sol qui domine. Il y a donc dans le caractère frappant des sapins obliques une composante intellectuelle, un savoir. Mais il y a néanmoins indubitablement une composante physiologique.
Les données sur le mal des astronautes, à qui voir à travers un hublot du satellite l'horizon terrestre la terre en haut peut provoquer la nausée, montrent à quel point la continuité du champ vectoriel de la verticale est chez l'homme une nécessité (dans cet esprit, il serait intéressant de vérifier si l'illusion des « sapins obliques» se présente avec la même netteté lorsque l’observateur est couché sur la banquette du compartiment...). Nous usons ici, librement, de ce terme de référentiel pour évoquer les contraintes invariantes associées à un mode perceptif et au lieu sur lequel il s'exerce. Les lecteurs désireux d'en savoir plus pourront lire l'ouvrage de F. Gonseth.

\section{RÉFÉRENCES}

- Ferdinand Gonseth, Le Référentiel, univers obligé de médiatisation, Lausanne, L'Âge d'Homme, 1980.

- C.-A. Lapparent (de), La Géologie en chemin de fer, description géologique du Bassin parisien et des régions adjacentes, Paris, F. Savy, 1888.

- René Thom, Apologie du Logos, Paris, Hachette, 1990.

- E.-C Zeeman, «Sudden Changes of Perception» (« Changements brusques de la perception ", in Jean Petitot (dir.), Logos et Théorie des catastrophes, Colloque de Cerisy, 1982, Genève, Patino, mars 1989. 


\section{DISCUSSIONS}

Discussions, sous la présidence de M. Barry Bergdoll, professeur à l'université de Columbia (New York, États-Unis).

\section{Yves Machefert-Tassin}

Comment introduisez-vous la vitesse dans ces référentiels?

\section{René Thom}

Je ne l'introduis pas directement; elle joue un rôle bien entendu dans la nature des référentiels choisis : comme on l'a fait observer, plus on va vite, plus il faut un gros référentiel. Si on va lentement on peut prendre un référentiel plus petit, plus proche. À part cela, je ne sais pas s'il y a un facteur de choix plus spécifique.

\section{Jacques Guillerme}

Tout le monde connait l'effet étrange introduit par la vitesse : l'accommodation « travaille » entre 0 et 50 mètres; dans ces limites le terrain vu du train semble nous fuir. Si, d'un seul coup, on porte le regard à l'horizon, par exemple sur la crête de collines, notre regard semble les accompagner. Il y a donc une zone d'inversion phénoménale dont je me demande à quoi elle peut correspondre du point de vue psycho-physiologique et s'il y a là un rapport quelconque avec la zone en pointillés du diagramme que vous nous avez montré.

\section{René Thom}

Faites-vous allusion au phénomène familier de notre enfance : quand on regardait par la portière les fils du télégraphe qui accompagnait la voie, on les voyait monter et descendre. Est-ce un phénomène du même genre?

\section{Jacques Guillerme}

Je ne disqualifierais pas cette observation, qui rencontre la coalescence routinière de perceptions qui ont pour lieu commun le sujet percevant.

\section{René Thom}

Il me semble clair que les objets proches, quand nous les identifions, exigent que nous déplacions dans une certaine mesure notre fovea, l'axe central de l'œil. Et ceci par une vitesse ambulée qui est évidemment d'autant plus grande que l'objet est plus proche. Si en revanche vous regardez un objet très loin, alors vous pouvez en quelque sorte le fixer et par un déplacement très lent le maintenir très longtemps. Vous pensez qu'on peut l'interpréter en terme de gradient vis-à-vis du sujet? Cela me semble possible. Les objets proches sont toujours examinés dans une optique de défense de l'ego. Tandis que les objets lointains sont considérés comme appartenant, précisément, au lointain, c'est-à-dire ne nous importent biologiquement que très peu. Il est donc possible qu'il y ait un facteur de ce genre. 